\title{
Apply Cross Spectral Analysis to Transfer Function Model Identification
}

\author{
Zheng Changjiang ${ }^{1,2}$ \\ ${ }^{1}$ College of Humanities, Shanghai Institute of \\ Technology \\ ${ }^{2}$ An Tai College of Economics \& management, \\ Shanghai Jiao Tong University \\ Shanghai, China \\ Chen Qifang \\ State Grid Shanghai \\ Shanghai China
}

\author{
Tong Linbai \\ Shanghai Jiulong Management Consulting Co.LTD \\ Shanghai, China \\ Cui Youxiang* \\ An Tai College of Economics \& management, Shanghai \\ Jiao Tong University \\ Shanghai, China \\ *Corresponding author \\ E-mail: cuiyouxiang@aliyun.com
}

\begin{abstract}
Transfer function models is of considerable interest in economics, engineering, biology, and many other fields. Models of this kind can describe not only the behavior of industrial processes but also that of economic and business systems. Transfer function model building is important because it is only when the dynamic characteristics of a system are understood that intelligent direction, manipulation, and control of the system is possible. Engineering methods for estimating transfer functions are usually based on the choice of special inputs to the system such as step and sine wave inputs and "pulse" inputs. These methods have been useful when the system is affected by small amounts of noise but are less satisfactory otherwise. In the presence of appreciable noise, it is necessary to use statistical methods for estimating the transfer function. In this paper we show that an alternative method for identifying transfer function models, which does not require prewhitening of the input, can be based on spectral analysis. Furthermore, it is easily generalized to multiple inputs.
\end{abstract}

Keywords- cross spectrum; transfer function models; frequency response function; cross spectral analysis; Fourier transform

\section{INTRODUCTION}

A topic of considerable industrial interest is the study of process dynamics. Such a study is made (1) to achieve better control of existing plants and (2) to improve the design of new plants. In particular, several methods have been proposed for estimating the transfer function of plant units from process records consisting of an input time series $X_{t}$ and an output time series $Y_{t}$.

As shown in Fig .1, where the input $X_{t}$ and the output $Y_{t}$. A hypothetical impulse response function $v_{j}, j=0,1$, $2, \ldots$, which determines the transfer function for the system through a dynamic linear relationship between input $X_{t}$ and output $Y_{t}$ of the form

$$
Y_{t}=\sum_{j=0}^{\infty} v_{j} X_{t-j}
$$

is also shown in the figure as a bar chart. Transfer function models that relate an input process $\mathrm{X}_{\mathrm{t}}$ to an output process $\mathrm{Y}_{\mathrm{t}}$ are introduced in this paper and many of their properties are examined.

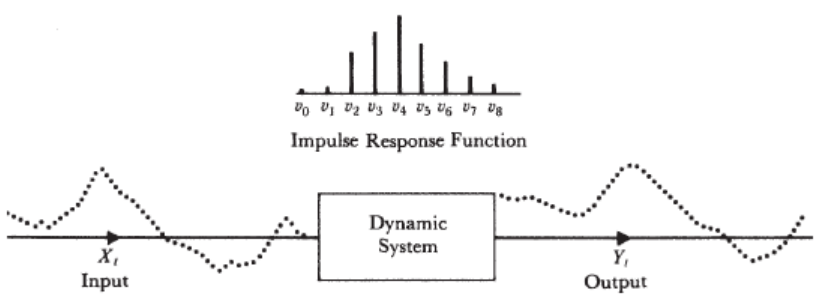

Figure 1. Input and output time series in relation to a dynamic system

With suitable inputs and outputs, the dynamic system of Fig .1 might represent an industrial process, the economy of a country, or the behavior of a particular corporation or government department.

In engineering, a transfer function (also known as the system function or network function and, when plotted as a graph, transfer curve) is a mathematical representation for fit or to describe inputs and outputs of black box models.

Technically it is a representation in terms of spatial or temporal frequency, of the relation between the input and output of a linear time-invariant system with zero initial conditions and zero-point equilibrium. With optical imaging devices, for example, it is the Fourier transform of the point spread function (hence a function of spatial frequency) i.e. the intensity distribution caused by a point object in the field of view.

Transfer functions are commonly used in the analysis of systems such as single-input single-output filters, typically within the fields of signal processing, communication theory, and control theory. The term is often used exclusively to refer to linear, time-invariant systems (LTI), as covered in this article. Most real systems have non-linear input/output characteristics, but many systems, when operated within nominal parameters (not "over-driven") have behavior that is close enough to linear that LTI system theory is an acceptable representation of the input/output behavior. 
In time series analysis, the cross-spectrum is used as part of a frequency domain analysis of the crosscorrelation or cross-covariance between two time series.

\section{GENERAL CONSIDERATIONS IN IDENTIFYING TRANSFER FUNCTION MODELS}

Some general remarks can be made concerning the procedure for identifying transfer function and noise models that just described

1. For many practical situations, when the effect of noise is appreciable, a delayed first- or second-order system or some simplification of it, would often provide as elaborate a model as could be justified for the data. In practice, the output $\mathrm{Y}$ could not be expected to follow exactly the pattern determined by the transfer function model, even if that model were entirely adequate. Disturbances of various kinds other than $\mathrm{X}$ normally corrupt the system.

2. To start off the recursion we need to know certain initial values. This need is not, of course, a shortcoming of the method of calculation but comes about because with a transfer function model, the initial values of $\mathrm{Y}$ will depend on values of $\mathrm{X}$ that occurred before observation was begun. In practice, when the necessary initial values are not known, we can substitute mean values for unknown Y's and X's (zeros if these quantities are considered as deviations from their means). The early calculated values will then depend upon this choice of the starting values. However, for a stable system, the effect of this choice will be negligible after a period sufficient for the impulse response to become negligible.Efficient estimation is only possible assuming the model form to be known. The estimates given are in general necessarily inefficient therefore. They are employed at the identification stage because they are easily computed and can indicate a form of model worthy to be fitted by more elaborate means.

3. Even if these were efficient estimates, the number required to trace out the impulse response function fully would typically be considerably larger than the number of parameters in a transfer function model. In cases where the $\delta$ and $\omega$ in an adequate transfer function model could be estimated accurately, nevertheless, the estimates of the corresponding v's could have large variances and be highly correlated .

A nonlinear least squares algorithm, analogous to that given for fitting the stochastic model can be used to obtain the least squares estimates and their approximate standard errors. The algorithm will behave well when the sum-of-squares function is very roughly quadratic. However, the procedure can sometimes run into trouble, in particular if the parameter estimates are very highly correlated (if, e.g., the model approaches singularity due to near-common factors in the factorizations of the operators), or in some cases, if estimates are near a boundary of the permissible parameter space. In difficult cases the estimation situation may be clarified by plotting sums-of-squares contours for selected two-dimensional sections of the parameter space.

As with stochastic models, the derivatives may be computed recursively. However, it seems simplest to work with a standard nonlinear least squares computer program in which derivatives are determined numerically and an option is available of "constrained iteration" to prevent instability

4.Usually, only very rough estimates are possible with the available data. However, some kind of rudimentary modeling may be possible by postulating a plausible but simple transfer function/noise model, fitting directly by the least squares procedures, and applying diagnostic checks leading to elaboration of the model when this proves necessary.

5. Since rather simple transfer function models of first or second order, with or without delay, are often adequate, iterative model building should begin with a fairly simple model, looking for further simplification if this is possible, and reverting to more complicated models only as the need is demonstrated.

6. When simplification by factorization is possible, but is overlooked, the least squares estimation procedure may become extremely unstable since the minimum will tend to lie on a line or surface in the parameter space rather than at a point. Conversely, instability in the solution can point to the possibility of simplification of the model. One reason for carrying out the identification procedure before fitting the model is to avoid redundancy or, conversely, to achieve parsimony in parameterization.

\section{IDENTIFICATION PROCEDURE OF TRANSFER FUNCTION MODELS}

Suppose that the transfer function model

$$
Y_{t}=v(B) X_{t}+N_{t}
$$

Can be parsimoniously parameterized in the form

$$
Y_{t}=\delta^{-1}(B) \omega(B) X_{t-b}+N_{t}
$$

Where

$$
\begin{gathered}
\delta(B)=1-\delta_{1} B-\delta_{2} B^{2}-\cdots-\delta_{\tau} B^{\tau} \\
\omega(B)=\omega_{0}-\omega_{1} B-\omega_{2} B^{2}-\cdots-\omega_{s} B^{s}
\end{gathered}
$$

The identification procedure is as follows:

1. Derive rough estimates $\widehat{v}_{J}$ of the impulse response weights $v_{j}$ in (2).

2 . Use the estimates $\widehat{v}_{j}$ so obtained to make guesses of the orders $r$ and $s$ of the denominator and numerator operators in (3) and of the delay parameter $b$.

3. Substitute the estimates $\widehat{v}_{j}$ in the equations with values of $r, s$, and $b$ obtained from initial estimates of the parameters $\delta$ and $\omega$ in (3).

Knowing the $\widehat{v}_{J}$, values of $b, r$, and $s$ may be determined by using the following facts established in the 
model of the form of (3) the impulse response weights vj consist of:

1. $b$ zero values $v_{0}, v_{l}, \ldots, v_{b-1}$

2. A further $s-r+1$ values $v_{b}, v_{b+l}, \ldots, v_{b+s-r}$ following no fixed pattern

(no such values occur if $s<r$ ).

3. Values $v_{j}$ with $j \geqslant b+s-r+1$ that follow the pattern dictated by an rth-order difference equation that has $r$ starting values $v_{b+s}, \ldots, v_{b+s-r+1}$. starting values $v_{j}$ for $j$ $<b$ will, of course, be zero.

The basic tool that is employed here in the identification procedure is the cross-correlation function between input and output.

When the processes are nonstationary, it is assumed that stationarity can be induced by suitable differencing. Nonstationary behavior is suspected if the estimated autoand cross-correlation functions of the $\left(X_{t}, Y_{t}\right)$ series fail to damp out quickly. It assume that a degree of differencing $\mathrm{d}$ necessary to induce stationarity has been achieved when the estimated auto- and cross correlations $\mathrm{r}_{\mathrm{xx}}(\mathrm{k}), \mathrm{r}_{\mathrm{yy}}(\mathrm{k})$, and $\mathrm{r}_{\mathrm{xy}}(\mathrm{k})$ of $\mathrm{x}_{\mathrm{t}}=\nabla^{\mathrm{d}} \mathrm{X}_{\mathrm{t}}$ and $\mathrm{y}_{\mathrm{t}}=\nabla^{\mathrm{d}} \mathrm{Y}_{\mathrm{t}}$ damp out quickly. In practice, $\mathrm{d}$ is usually 0,1 , or 2 .

\section{IDENTIFICATION OF SINGLE INPUT TRANSFER FUNCTION MODELS TYPE}

Suppose that the transfer function $v(B)$ is defined so as to allow the possibility of nonzero impulse response weights $v_{j}$ for $j$ a negative integer, so that

$$
\mathrm{v}(\mathrm{B})=\sum_{k=-\infty}^{\infty} v_{k} B^{k}
$$

To the general case, suppose that (where necessary, after suitable differencing) the transfer function-noise model could be written

$$
y_{t}=v(B) x_{t}+n_{t}
$$

The Impulse Response Function without Prewhitening can be written in the form

$$
\gamma_{x y}(k)=\sum_{j=-\infty}^{\infty} v_{j} \gamma_{x x}(k-j) \quad k=0, \pm 1, \pm 2, \cdots
$$

Cross-covariance generating function can be defined as fellow

$$
\gamma^{x y}(k)=\sum_{j=-\infty}^{\infty} \gamma_{x y}(k) B^{k}
$$

On multiplying throughout in (4) by $B^{k}$ and summing, we obtain

$$
\gamma^{x y}(k)=v(B) \gamma^{x x}(B)
$$

Substitute B $=e^{-i 2 \pi f}$ in (7), we can obtain the cross spectrum $p_{x y}(f)$ between input $x_{t}$ and output $y_{t}$. Making the same substitution in (8) gets

$$
v\left(e^{-i 2 \pi f}\right)=\frac{p_{x y}(f)}{p_{x x}(f)} \quad-\frac{1}{2} \leq f \leq \frac{1}{2}
$$

Where

$$
v\left(e^{-i 2 \pi f}\right)=G(f) e^{-i 2 \pi \varphi(f)}=\sum_{k=-\infty}^{\infty} v_{k} e^{-i 2 \pi f k}
$$

is called the frequency response function of the system transfer function relationship and is the Fourier transform of the impulse response function.

Since $v\left(e^{-i 2 \pi f}\right)$ is complex valued, it can be written as a product involving a gain functionG(f $)=\left|v\left(e^{-i 2 \pi f}\right)\right|$ and a phase function $\varphi(f)$. Equation (9) shows that the frequency response function is the ratio of the cross spectrum to the input spectrum.

Knowing $v\left(e^{-i 2 \pi f}\right)$, the impulse response function $v_{k}$ can then be obtained from

$$
v_{k}=\int_{-1 / 2}^{1 / 2} v\left(e^{-i 2 \pi f}\right) e^{i 2 \pi f k} d f
$$
is

The autocovariance generating function of the noise $n_{t}$

$$
\gamma^{n n}(B)=\gamma^{y y}(B)-\frac{\gamma^{x y}(B) \gamma^{x y}(F)}{\gamma^{x x}(B)}
$$

On substituting $\mathrm{B}=e^{-i 2 \pi f}$ in (10), we obtain the expression

$$
p_{n n}(f)=p_{y y}(f)\left[1-k_{x y}^{2}(f)\right]
$$

For the spectrum of the noise process, where

$$
k_{x y}^{2}(f)=\frac{\left|p_{x y}(f)\right|^{2}}{p_{x x}(f) p_{y y}(f)}
$$

and $k_{x y}(f)$ is the coherency spectrum between the series $x_{t}$ and $y_{\mathrm{t}}$. The coherency spectrum $k_{x y}(f)$ at each frequency $\mathrm{f}$ behaves like a correlation coefficient between the random components at frequency $f$ in the spectral representations of $x_{t}$ and $y t$. Knowing the noise spectrum, the noise autocovariance function $\gamma_{n n}(k)$ may then be obtained from

$$
\gamma_{n n}(k)=2 \int_{0}^{1 / 2} p_{n n}(f) \cos (2 \pi f k) d f
$$

By substituting estimates of the spectra, estimates of the impulse response weights $v_{k}$ and noise autocorrelation function are obtained.

These can be used to identify the transfer function model and noise model.

\section{IDENTIFICATI ON OF MULTIPLE INPUT TRANSFER FUNCTION MODELS}

Generalize the model

$$
Y_{t}=v(B) X_{t}+N_{t}=\delta^{-1}(B) \omega(B) X_{t-b}+N_{t}
$$

to allow for several inputs $\mathrm{X}_{1, \mathrm{t}}, \mathrm{X}_{2, \mathrm{t}}, \ldots, \mathrm{X}_{\mathrm{m}, \mathrm{t}}$. Thus 


$$
\begin{gathered}
Y_{t}=v_{1}(B) X_{1, t}+\cdots+v_{m}(B) X_{m, t}+N_{t} \\
Y_{t}=\delta_{1}^{-1}(B) \omega_{1}(B) X_{1, t-b}+\cdots+\delta_{m}^{-1}(B) \omega_{m}(B) X_{m, t-b} \\
+N_{t}
\end{gathered}
$$

Where $v_{\mathrm{j}}(B)=\delta_{\mathrm{j}}^{-1}(B) \omega_{1} B^{b_{j}}$ is the generating function of the impulse response weights relating $X_{j, t}$ to the output $Y_{t}$. We assume, as before, that after differencing,(14) may be written

$$
y_{t}=v_{1}(B) x_{1, t}+\cdots+v_{m}(B) x_{m, t}+n_{t}
$$

Where $y_{t}, x_{1, t}, \cdots, x_{m, t}$ and $n_{t}$ are all jointly stationary processes. Multiplying throughout by $x_{1, t-k}, x_{2, t-k}, \cdots, x_{m, t-k}$ in turn, taking expectations and forming the generating functions, we obtain

$$
\begin{aligned}
& \gamma^{x_{1} y}=v_{1}(B) \gamma^{x_{1} x_{1}}(B)+v_{2}(B) \gamma^{x_{1} x_{2}}(B)+\cdots \\
& +v_{m}(B) \gamma^{x_{1} x_{\mathrm{m}}}(B) \\
& \gamma^{x_{2} y}=v_{1}(B) \gamma^{x_{2} x_{1}}(B)+v_{2}(B) \gamma^{x_{2} x_{2}}(B)+\cdots \\
& +v_{m}(B) \gamma^{x_{2} x_{\mathrm{m}}}(B) \\
& \vdots \quad \vdots \quad \vdots \\
& \gamma^{x_{m} y}=v_{1}(B) \gamma^{x_{m} x_{1}}(B)+v_{2}(B) \gamma^{x_{m} x_{2}}(B) \\
& +\cdots+v_{m}(B) \gamma^{x_{m} x_{\mathrm{m}}(B)}
\end{aligned}
$$

On substituting $\mathrm{B}=e^{-i 2 \pi f}$, the spectral equations are obtained. For example, with $\mathrm{m}=2$,

$$
\begin{aligned}
& p_{x_{1}, y}(f)=H_{1}(f) p_{x_{1} x_{1}}(f)+H_{2}(f) p_{x_{1} x_{2}}(f) \\
& p_{x_{2}, y}(f)=H_{1}(f) p_{x_{2} x_{1}}(f)+H_{2}(f) p_{x_{2} x_{2}}(f)
\end{aligned}
$$

\section{ACKNOWLEDGMENT}

This research was financially supported by the National Science Foundation of China (71373158) and the National Social Sciences Fund of China (12AZD015).

\section{REFERENCES}

[1] Box G E P, Jenkins G M, Reinsel G C. Time series analysis forecasting and control[M]. Wiley. com, 2013.

[2] Youxiang C, Wei H, Jianshe L, et al. Feedforward Control Analytical Model in SPC[J]. 2014.

[3] Mao Z, Todd M. Statistical modeling of frequency response function estimation for uncertainty quantification[J]. Mechanical Systems and Signal Processing, 2013, 38(2): 333-345.

[4] Hannan, E. J. and M. Deistler, The Statistical Theory of Linear Systems, Wiley, New

[5] Hannan, E. J., W. M. Dunsmuir, and M. Deistler, "Estimation of vector ARMAX models,” J. Multivariate Anal., 10, 275-295, 1979.

[6] Reinsel, G., "Maximum likelihood estimation of stochastic linear difference equations with autoregressive moving average errors," Econometrica, 47, 129-151, 1979.

[7] You-xiang C, Jian-xin Y, Feng L. Applying fast Fourier transform (FFT) to statistic quality control[C]//Industrial Engineering and Engineering Management (IEEM), 2010 IEEE International Conference on. IEEE, 2010: 2144-2148. 57670.

[8] Brillinger D R. The identification of a particular nonlinear time series system[M]//Selected Works of David Brillinger. Springer New York, 2012: 607-613.

[9] Friston K J, Bastos A, Litvak V, et al. DCM for complex-valued data: cross-spectra, coherence and phase-delays[J]. Neuroimage, 2012, 59(1): 439-455.

[10] Bendat J S, Piersol A G. Random data: analysis and measuremen procedures[M]. John Wiley \& Sons, 2011.

[11] Coles W, Hobbs G, Champion D J, et al. Pulsar timing analysis in the presence of correlated noise[J]. Monthly Notices of the Royal Astronomical Society, 2011, 418(1): 561-570.

[12] Alink M S O, Klumperink E A M, Kokkeler A B J, et al. A CMOScompatible spectrum analyzer for cognitive radio exploiting crosscorrelation to improve linearity and noise performance $[\mathrm{J}]$. Circuits and Systems I: Regular Papers, IEEE Transactions on, 2012, 59(3): 479-492.

[13] Keesman K J. System identification: an introduction[M]. Springer, 2011.

[14] Chatfield C. The analysis of time series: an introduction[M]. CRC press, 2013.

[15] Mao Z, Todd M. A model for quantifying uncertainty in the estimation of noise-contaminated measurements of transmissibility[J]. Mechanical Systems and Signal Processing, 2012, 28: 470-481.

[16] Challinor A, Lewis A. Linear power spectrum of observed source number counts[J]. Physical Review D, 2011, 84(4): 043516. 